\title{
Evaluation of general nutrition knowledge in elite Australian athletes
}

\author{
Jessica K. Spendlove ${ }^{1}$, Susan E. Heaney ${ }^{2}$, Janelle A. Gifford ${ }^{1}$, Tania Prvan $^{3}$, Gareth S. Denyer ${ }^{1}$ \\ and Helen T. O'Connor ${ }^{2 *}$ \\ ${ }^{1}$ Faculty of Science, University of Sydney, Sydney, NSW, Australia \\ ${ }^{2}$ Discipline of Exercise and Sport Science, Faculty of Health Science, University of Sydney (Cumberland Campus), \\ PO Box 170, Lidcombe, NSW 1825, Australia \\ ${ }^{3}$ Faculty of Science, Macquarie University, Sydney, NSW, Australia
}

(Submitted 28 February 2011 - Final revision received 1 August 2011 - Accepted 16 August 2011 - First published online 10 October 2011)

\section{Abstract}

The aim of the present study was to investigate and benchmark the level of general nutrition knowledge in elite Australian athletes (EA) against a similar aged community (CM) and criterion sample with dietetic training (DT). EA ( $n$ 175), CM ( $n$ 116) and DT ( $n$ 53) completed the General Nutrition Knowledge Questionnaire (GNKQ), which assesses four domains (sections A-D) of general nutrition knowledge (section A: dietary guidelines; section B: sources of nutrients; section C: choosing everyday foods; section D: diet-disease relationships). Age, sex and education level were collected in all groups, and athletic calibre and sport type (team or individual) in EA. Dietitians and nutrition scientists ( $n$ 53) re-examined the GNKQ for content validity, resulting in instrument revision (R-GNKQ; ninety-six items). Psychometric assessment (internal consistency: Cronbach- $\alpha$; test-retest: Spearman rank correlation) was performed in a sub-sample ( $n$ 28). Independent $t$ tests, ANOVA and ANCOVA ( $\chi^{2}$ for categorical variables) were used to assess between-group differences. DT scored higher than EA and $\mathrm{CM}$ in all sub-sections and overall $(P<0 \cdot 005)$. EA scored lower than CM in GNKQ for section $\mathrm{B}(P<0 \cdot 005)$ and overall $(P<0.005)$, and in R-GNKQ for section B $(P<0.005)$, section C $(P<0.005)$, section D $(P=0.006)$ and overall $(P<0 \cdot 005)$. Overall score was influenced by age $(P=0.036$ for GNKQ: $P=0.053$ for R-GNKQ), sex $(P=0.016$ for GNKQ: $P=0.003$ for R-GNKQ) and athletic calibre ( $P=0.029$ for R-GNKQ only), but not level of education, living situation or ethnicity. EA and CM performed best on section A and worst on D. EA had lower overall general knowledge scores than CM. This was significantly influenced by age and sex.

\section{Key words: Nutrition knowledge: Athletes: Nutrition education}

Meeting energy and nutrient needs is a priority for athletes to facilitate achievement of peak performance ${ }^{(1)}$. Although athletes have special nutritional needs, the basis of all athlete nutrition is a healthy, well-balanced diet. Although a specific set of dietary recommendations does not exist for athletes, it is recognised that, at a minimum, athletes need to consume a diet consistent with recommendations for the general population for overall good health.

One of the primary strategies for assisting athletes to consume an adequate diet is the provision of nutrition education. The aim of this education is to address the need for athletes to know what to eat and how to select, and prepare a wide variety of foods necessary for a healthy diet. The underlying assumption is that adequate nutrition knowledge, appropriate food selection and preparation skills are needed for better dietary intake, and that education in these areas will carry through to behavioural change. Although it is acknowledged that adequate nutrition knowledge may not always translate into appropriate dietary practice, it has been argued that even a small amount of nutrition knowledge is pivotal in supporting the adoption of healthier food habits ${ }^{(2)}$. Individuals with higher nutrition knowledge are almost twenty-five times more likely to meet present recommendations for fruit, vegetable and fat intake than those with low knowledge ${ }^{(3)}$. Although known practical barriers may inhibit implementation of appropriate dietary strategies in athlete groups ${ }^{(4)}$, there is limited information about the nutrition knowledge of athletes and the influence this has on dietary intake ${ }^{(5)}$.

A recent systematic review of studies in athletes ${ }^{(5)}$ reported a weak, positive association between nutrition knowledge and better dietary intake in five of nine papers exploring this relationship. Unfortunately, many of the available studies failed to measure either nutrition knowledge or dietary

Abbreviations: CM, community; DT, dietetic-trained; EA, elite Australian athletes; GNKQ, General Nutrition Knowledge Questionnaire; R-GNKQ, reexamined General Nutrition Knowledge Questionnaire; SES, socioeconomic class.

*Corresponding author: Dr H. T. O'Connor, fax +61293519204, email helen.oconnor@sydney.edu.au 
intake with sufficient rigour. Similar to athlete studies, a number of methodological limitations with the instruments measuring nutrition knowledge, including the representativeness of the sample, may in part explain why the link between nutrition knowledge and dietary intake in the general community is also weak ${ }^{(6,7)}$.

Nutrition knowledge is a difficult construct to measure. Instruments may probe knowledge about functions of nutrients, their relevance to health, food sources or how to purchase, plan and prepare a diet that is healthy, nutritious and suitable to individual needs. Nutrition knowledge encompasses what has been termed declarative knowledge of facts (e.g. food sources of protein) and procedural knowledge (e.g. how to plan, purchase and prepare food to make up a diet that has sufficient protein $)^{(2)}$. Strong declarative knowledge without procedural skills may not translate to healthier dietary intake. Nutrition knowledge is also influenced by beliefs about food and nutrition, which may not be evidenced based but rather steeped in cultural or present secular thinking ${ }^{(8)}$. Conflicting and evolving nutrition research, food product advertising, the complexity of behaviours needed to achieve healthy eating and strong media and Internet coverage of nutrition issues make it challenging for clear nutrition messages to translate at a population level.

Numerous studies have identified demographic factors that influence nutrition knowledge, including sex, age, sociodemographic status and level of education ${ }^{(3,7,9-13)}$. Studies reporting the influence of age consistently find younger adult participants score lower than middle-aged individuals ${ }^{(3,10,14)}$. This has been demonstrated in both athlete ${ }^{(14)}$ and general community samples ${ }^{(3,10,11)}$. Sex is also important, with males usually scoring lower than females in both community $^{(3,7,10-12)}$ and athlete samples ${ }^{(14-18)}$. Socioeconomic class (SES) and/or level of education has been associated with superior performance on a number of other nutrition knowledge instruments, with higher education positively associated with knowledge in both community ${ }^{(7,9-13)}$ and athlete cohorts ${ }^{(19,20)}$. In sports-specific studies, additional factors including athletic calibre, sport type and emphasis on physique, which can increase focus on dietary intake, may also influence nutrition knowledge. There is some evidence supporting both athletic calibre ${ }^{(18,21)}$ and participation in physique-oriented sports making a positive impact nutrition knowledge scores ${ }^{(16)}$. However, most studies conducted in athlete populations fail to assess these parameters or other potential sport-specific confounders (e.g. team $v$. individual sports). This, together with the use of inadequately validated instruments, limits the capacity to evaluate the level of nutrition knowledge and the impact of sports-specific factors in athlete cohorts.

The present study aims to use a previously validated instrument ${ }^{(22,23)}$ to investigate and benchmark the level of general nutrition knowledge in elite Australian athletes against a similar aged, non-athlete community sample and a dietetic-trained cohort with high-level expertise in nutrition. A secondary aim of the present study was to further evaluate the validity and psychometric properties of the instrument in this cohort and investigate the impact of previously identified confounders including age, sex, level of education, living situation, country of birth, first language and, in the athletes, sport type and athletic calibre on nutrition knowledge.

\section{Methods}

\section{Participants}

Three groups including elite Australian athletes (EA), a community (CM) sample and a dietetic-trained (DT) cohort with high-level nutrition expertise were recruited to complete the General Nutrition Knowledge Questionnaire (GNKQ) ${ }^{(11,14)}$. The EA group included scholarship holders from four (nonresidential) Australian state institutes or academies of sport, elite competitors from an Australian life-saving club and a youth $(<20$ years $)$ team competing in the National Rugby League competition. To obtain a scholarship at the state institutes or academies of sport, athletes were typically representative at national or international level. To benchmark EA scores, $\mathrm{CM}$ and DT cohort samples were recruited. The CM sample included Faculty of Arts University students and members of Scouts Australia (Scouts) aged 18-26 years. The DT sample were Australian undergraduate and postgraduate dietetic interns completing the final semester of University training and Australian Accredited Practicing Dietitians recruited on the basis of extensive experience in nutrition and dietetics. The CM cohort was expected to provide a suitable comparison group to the EA with respect to age and sex, with the Faculty of Arts students tertiary educated. The DT cohort was included as the benchmark standard for the EA and CM scores to be measured against. Recruitment was through flyers or electronic newsletters. Data were collected between the years 2009 and 2010. The present study was conducted according to the guidelines laid down in the Declaration of Helsinki, and all procedures involving human subjects/patients were approved by the University of Sydney, Human Ethics Committee (HREC no. 9640). Written consent was obtained from all subjects/patients.

\section{General Nutrition Knowledge Questionnaire}

The GNKQ has been previously well validated and used to measure general nutrition knowledge in a large UK community sample ${ }^{(22)}$, athlete samples in the $\mathrm{UK}^{(24)}$ and the $\mathrm{USA}^{(17)}$ and an Australian community sample ${ }^{(23)}$. When the GNKQ was initially constructed, it was also psychometrically validated. The questionnaire successfully measured a spectrum of nutrition knowledge (content validity), and when administered to cohorts with varying degrees of nutrition knowledge, significantly different scores were achieved (construct validity). Internal consistency and test-re-retest reliability were also measured $^{(25)}$. Minor modifications for the Australian population were made to the UK instrument to incorporate Australian terminology used for some foods rather than common UK food names ${ }^{(23)}$. Examples included replacing the term 'calories' with 'kilojoules', 'orange squash' with '35\% orange juice' and 'luncheon meat' with 'lunch/sandwich meat'(23). Three additional items were included that covered key 
Australian public health messages and common misconceptions, resulting in a maximum of 113 points compared to the original version that contained 110 points ${ }^{(23)}$. The GNKQ probes a variety of nutrition knowledge domains, including dietary guideline recommendations (section A), nutrient content of foods (section B), everyday food choices (section C) and links between diet and disease (section D). Demographic characteristics including age, sex, level of education and ethnicity are captured. Some additional demographic questions to assess the level of physical activity, classified according to Australian public health guidelines, were added for the present study. Activity of more than $150 \mathrm{~min} /$ week was required to be classified as sufficient ${ }^{(26)}$. Additional questions relevant only to the EA group were used to collect information about hours spent training, type of sport and level of sports representation (state, national or international). The instrument contained a total of forty-five items assessing nutrition knowledge that were distributed over four sections: section A (four items: thirteen points), section B (twenty-one items: seventy points), section $\mathrm{C}$ (ten items: ten points) and section $\mathrm{D}$ (ten items: twenty points). The GNKQ featured different types of question styles, including multiple choice, yes/no or agree/ disagree items and questions that required participants to make food choices (e.g. select foods containing high or low amounts of fibre or saturated fat). Items probing diet-disease relationships were open ended and required participants to nominate diseases associated with diet-related lifestyle factors.

\section{Further validation of the General Nutrition Knowledge Questionnaire}

Further psychometric evaluation and content validation of the GNKQ was undertaken to examine its performance in this cohort. Personnel with high expertise in nutrition ( $n$ 53), including Accredited Practising Dietitians and nutrition researchers (expert panel), were asked to evaluate each item of the GNKQ using a purpose-developed evaluation form that captured opinion on whether each item should be retained, modified or deleted with an optional open-ended response to strengthen content validity. Feedback provided was evaluated by the research team (J. K. S., H. T. O'C., J. A. G., G. S. D. and S. E. H.) to establish consensus. When more than $50 \%$ of respondents in the DT sample answered an item incorrectly and it was also identified by the expert panel as unclear, ambiguous or inaccurate, the item was eliminated from analysis and scores for each sub-section and total were subsequently recalculated.

Modification to the GNKQ was termed as R-GNKQ. The R-GNKQ scores were calculated by omitting items from the GNKQ that were deemed ambiguous or inaccurate by the expert panel. All participants were therefore included in the analysis of both versions of the instrument. This recalculation as R-GNKQ resulted in a reduction of items in each section of the GNKQ (section A: thirteen reduced to eleven points; section B: seventy reduced to sixty-two points; section $\mathrm{C}$ : ten reduced to five points; section $\mathrm{D}$ : twenty reduced to eighteen points). Section D on diet-disease relationships required participants to list diseases associated with specific nutrient intake (e.g. saturated fat associated with heart disease). In the R-GNKQ, participants nominating one correct diet-disease relationship were allocated one point, with no additional score for further responses. This revised scoring system was based on feedback from the expert panel, which indicated that the number of responses required for each open-ended diet-disease relationship item was not clear. Additional diet-disease relationships, other than those featured in the original scoring system, were also accepted if they were evidence based and deemed appropriate by the research team. Scoring of two items (6 and 7) in section D was also amended to award one point for a correct response; hence, these items were weighted similarly to others in the instrument. This change was directed by feedback from the expert panel, which indicated that the dissimilar weighting of these items was inappropriate.

\section{Data collection and statistical analysis}

The GNKQ was self-administered on-line, and responses were directly recorded into a database. Differences between knowledge scores (total and sub-scores on the four sections of the instrument) were assessed using independent two-sample $t$ tests and one-way ANOVA. ANCOVA was used to investigate the impact of known and suspected confounders (age, sex, level of education, living situation, first language, country of birth, level of physical activity and, in addition, athletic calibre and sport type in EA). $\chi^{2}$ Analysis was used to evaluate baseline differences in categorical variables.

Internal consistency of the instrument was measured using Cronbach- $\alpha$ constructs, which evaluates how consistently items within each section of the instrument and overall score assess knowledge. Cronbach- $\alpha$ values range from 0 to 1 , with this scale indicating the consistency of responses. A score of 0.7 or greater is considered satisfactory ${ }^{(25)}$. Spearman rank correlation was used to assess the test-retest reliability on sub-samples of the EA $(n$ 17) and DT ( $n$ 11) who agreed to complete the instrument twice, 2 weeks apart. Participants were blind to the request for a retest when they completed the instrument on the first occasion.

Significance was set at $P<0 \cdot 05$, except when several variables (four sub-sections and overall score) were examined on the same participants. In this case, a Bonferroni correction was used and significance was set at $P<0 \cdot 01$. When sub-section and overall nutrition knowledge score were not normally distributed, data were transformed. Data were analysed using SPSS (version 16.0; SPSS, Chicago, IL, USA). All data were reported as mean values and standard deviations.

\section{Results}

\section{Demographic characteristics}

Participant characteristics are summarised in Table 1. Before pooling the scores from the Faculty of Arts students and Scouts (constituting the CM group), independent two-sample $t$ tests were performed on total and all four instrument sub-sections to check for significant differences. As no 
significant differences were found, data were pooled for analysis. A similar process was used to compare the dietetic interns and Accredited Practising Dietitians, who constituted the DT cohort. No significant differences were found in sub-section or total scores, hence the data were pooled for analysis.

Of the 344 participants completing the instrument, EA comprised $50.9 \%$ ( $n$ 175), the CM sample $33.7 \%(n$ 116) and the DT cohort $15.4 \%$ ( $n$ 53). There was a significant difference in sex $(P=0.006)$ between EA and CM groups, and the majority of participants in each cohort were female (EA: 56.6\%; CM: $72.5 \%$; and DT: 86.8\%). The EA and CM cohorts were young (EA: 18.9 (SD 4.9) years; CM: 21.9 (SD 4.2) years) and age was slightly but significantly different $(P<0.005)$. Most of the EA participants were in the final 4 years of high school $(66.3 \%)$, the majority of whom were in the final 2 years (11 and 12: $49 \cdot 7 \%$ of EA cohort). Most of the CM group (92.2\%) and all of DT (100\%) were tertiary educated. The majority of EA were classified to be sufficiently physically active ( $>150 \mathrm{~min} /$ week) except one participant who was injured at the time of instrument completion.
Approximately, two-thirds (65.5\%) of the CM sample were sufficiently active according to Australian public health guidelines, as expected, and this difference in physical activity between EA and CM was statistically significant $(P<0.005)$. Most of the participants in EA and CM were from Western countries (Australia, UK or North America), a smaller proportion were from Asia (EA: 1.7\%; CM: 8.6\%) and the remainder (EA: $1.7 \%$; CM: $0.9 \%$ ) were from a variety of other countries. More than half the EA and CM samples were working in a full- or part-time or casual capacity (Table 1). Most of the EA (84.6\%) and CM (72.4\%) respondents lived with family or a partner or spouse. The EA cohort was predominantly comprised of participants playing team sports $(77 \cdot 1 \%)$, with a smaller number engaged in individual (22.9\%) sports. Most participated at national $(40 \cdot 1 \%)$ or junior international (31.4\%) level, with smaller numbers (18.0\%) only competing at state or open international level (10.5\%). Although predominantly Western $(73.6 \%)$, there was a greater proportion of Asian participants in the DT cohort compared to EA and CM

Table 1. Participant demographic characteristics

(Mean values and standard deviations, numbers and percentages)

\begin{tabular}{|c|c|c|c|c|c|c|c|}
\hline \multirow[b]{3}{*}{ Characteristic } & \multicolumn{6}{|c|}{ Total sample ( $n$ 344) } & \multirow{3}{*}{$\begin{array}{c}P \text { (comparison between } \\
\text { EA and CM group means) }\end{array}$} \\
\hline & \multicolumn{2}{|c|}{$\begin{array}{l}\mathrm{EA}(n 175) \\
(50.9 \%)\end{array}$} & \multicolumn{2}{|c|}{$\begin{array}{c}\mathrm{CM}(n 116) \\
(33.7 \%)\end{array}$} & \multicolumn{2}{|c|}{$\begin{array}{l}\text { DT }(n 53) \\
(15.4 \%)\end{array}$} & \\
\hline & $n$ & $\%$ & $n$ & $\%$ & $n$ & $\%$ & \\
\hline \multicolumn{8}{|l|}{ Age (years) } \\
\hline Mean & \multirow{2}{*}{\multicolumn{2}{|c|}{$\begin{array}{c}18.9 \\
4.9\end{array}$}} & \multicolumn{2}{|c|}{$\begin{array}{c}21.9 \\
4 . ?\end{array}$} & \multicolumn{2}{|c|}{$29 \cdot 1$} & 0.000 \\
\hline SD & & & & & & & \\
\hline$\leq 18$ & 117 & $66 \cdot 9$ & 8 & 6.9 & 0 & 0 & \\
\hline $19-24$ & 46 & $26 \cdot 3$ & 93 & $80 \cdot 2$ & 21 & $39 \cdot 7$ & \\
\hline$\geq 25$ & 12 & 6.9 & 15 & $12 \cdot 9$ & 32 & $60 \cdot 1$ & \\
\hline \multicolumn{8}{|l|}{ Sex } \\
\hline Male & 76 & 43.4 & 32 & 27.5 & 7 & $13 \cdot 2$ & 0.006 \\
\hline Female & 99 & $56 \cdot 6$ & 84 & $72 \cdot 5$ & 46 & $86 \cdot 8$ & \\
\hline \multicolumn{8}{|l|}{ Level of education } \\
\hline High school $†$ & 116 & $66 \cdot 3$ & 9 & 7.8 & 0 & 0 & 0.000 \\
\hline Tertiary & 59 & 33.7 & 107 & $92 \cdot 2$ & 53 & 100 & \\
\hline \multicolumn{8}{|c|}{ Level of physical activity (min/week) } \\
\hline$<150$ & 1 & 0.6 & 40 & 34.5 & 14 & $26 \cdot 4$ & 0.000 \\
\hline$>150$ & 174 & 99.4 & 76 & $65 \cdot 5$ & 39 & 73.6 & \\
\hline \multicolumn{8}{|l|}{ Ethnicity } \\
\hline Western‡ & 169 & $96 \cdot 6$ & 105 & 90.5 & 39 & $73 \cdot 6$ & 0.035 \\
\hline Asian & 3 & 1.7 & 10 & $8 \cdot 6$ & 14 & $26 \cdot 4$ & \\
\hline Other & 3 & $1 \cdot 7$ & 1 & 0.9 & 0 & 0 & \\
\hline \multicolumn{8}{|l|}{ Employment status } \\
\hline Working§ & 92 & $52 \cdot 6$ & 84 & $72 \cdot 4$ & 34 & $64 \cdot 2$ & 0.001 \\
\hline Not working & 83 & 47.4 & 32 & $27 \cdot 6$ & 19 & $35 \cdot 8$ & \\
\hline \multicolumn{8}{|l|}{ Living arrangement } \\
\hline Partner/spouse & 12 & 6.9 & 13 & $11 \cdot 2$ & 23 & 43.4 & 0.369 \\
\hline Family\| & 136 & $77 \cdot 7$ & 71 & $61 \cdot 2$ & 12 & $22 \cdot 6$ & \\
\hline Peers/flatmates & 11 & $6 \cdot 3$ & 22 & $19 \cdot 0$ & 14 & $26 \cdot 4$ & \\
\hline Alone & 4 & $2 \cdot 2$ & 0 & 0 & 1 & 1.9 & \\
\hline Boarding school & 1 & 0.6 & 7 & $6 \cdot 0$ & 0 & 0 & \\
\hline Other & 11 & $6 \cdot 3$ & 3 & 2.6 & 3 & $5 \cdot 7$ & \\
\hline
\end{tabular}

EA, elite Australian athletes; CM, community; DT, criterion.

* Baseline categorical data analysed by $\chi^{2}$ analysis, significance set at $P<0.05$.

$\dagger$ Includes participants in final 4 years of high school - year 8-12 (14-18 years).

$\ddagger$ Includes predominantly Australian respondents and a minority from North America and the UK.

$\S$ Includes full time, part time and casual employment.

\| Family includes away living with one or two adults (parents or guardians) and being billeted. 
$(P=0 \cdot 035)$. Most were working and living with a partner or family (Table 1).

\section{Nutrition knowledge score on the General Nutrition Knowledge Questionnaire}

Total and sub-section scores (Table 2) on the GNKQ demonstrated that EA and CM samples scored significantly less than the DT cohort in each sub-section and overall $(P<0.005)$. There was no significant difference between EA and $\mathrm{CM}$ for section A $(P=1 \cdot 000)$, section C $(P=0.083)$ or section D $(P=0 \cdot 017)$. However, a significant difference was found between the EA and CM groups for section B $(P<0.005)$, which probed knowledge on food sources of nutrients and total score $(P<0.005)$. The EA scored highest for section A, knowledge of dietary recommendations (65.4\%), and the CM sample scored marginally better for section B, food sources of nutrients $(67 \cdot 7 \%)$, closely followed by section A (65.4\%). Scores for the knowledge of diet-disease relationships (section D) were lowest in both EA (45.0\%) and CM (50.0\%) samples (Table 2). When compared to DT, EA scored substantially and significantly (all $P<0.005$ ) less in total score (mean difference $28.7 \%$ ) and in each sub-section of the instrument (mean difference for section A: $-12.5 \%$; section B: $-31.2 \%$; section C: $-16.5 \%$; and section $\mathrm{D}$ : $-43.5 \%$ ). A similar pattern was observed for CM group scores when compared to DT (mean difference for total score: $-23 \cdot 2 \%$; section A: $-12 \cdot 2 \%$; section B: $-24 \cdot 3 \%$; section $\mathrm{C}$ : $-12.3 \%$; and section $\mathrm{D}$ : $-37.5 \%)$. Where EA scored significantly lower than $\mathrm{CM}$, the mean difference was $6.9 \%$ for section B and 5.6\% less on total score.

\section{Nutrition knowledge scores on the re-examined General} Nutrition Knowledge Questionnaire

Overall and individual sub-section scores on the R-GNKQ tended to be higher for all groups compared with the GNKQ (Table 3). In the R-GNKQ, EA and CM scored significantly lower than the DT cohort on all sub-sections and overall nutrition knowledge score $(P<0 \cdot 005)$, similar to the GNKQ. As with the GNKQ, there was also no significant difference between EA and CM scores for section A $(P=0 \cdot 490)$. However, for all remaining sub-sections and the overall nutrition knowledge score, there was a significant difference between EA and CM, with EA scoring significantly lower than CM (Table 3). The EA $(73.6 \%)$ and CM (76.4\%) scored highest for section A, knowledge of dietary recommendations, and lowest for section D, knowledge of diet-disease relationships (EA: $48.9 \%$; CM: $55.6 \%$ ). When compared to DT, EA scored substantially and significantly (all $P<0.005$ ) less in total score (mean difference $-33.1 \%$ ) and in each sub-section of the instrument (mean difference for section A: $-11.4 \%$; section B: $-31.5 \%$; section C: $-26.8 \%$; and section $\mathrm{D}:-53.6 \%$ ). A similar pattern was observed for CM group scores when compared to DT (mean difference for total score: $-24.5 \%$; section A: $-9.3 \%$; section $\mathrm{B}:-24.4 \%$; section C: $-18.3 \%$; and section $\mathrm{D}:-35.2 \%)$. Where EA scored significantly lower than $\mathrm{CM}$, the mean difference was $-7.2 \%$ for

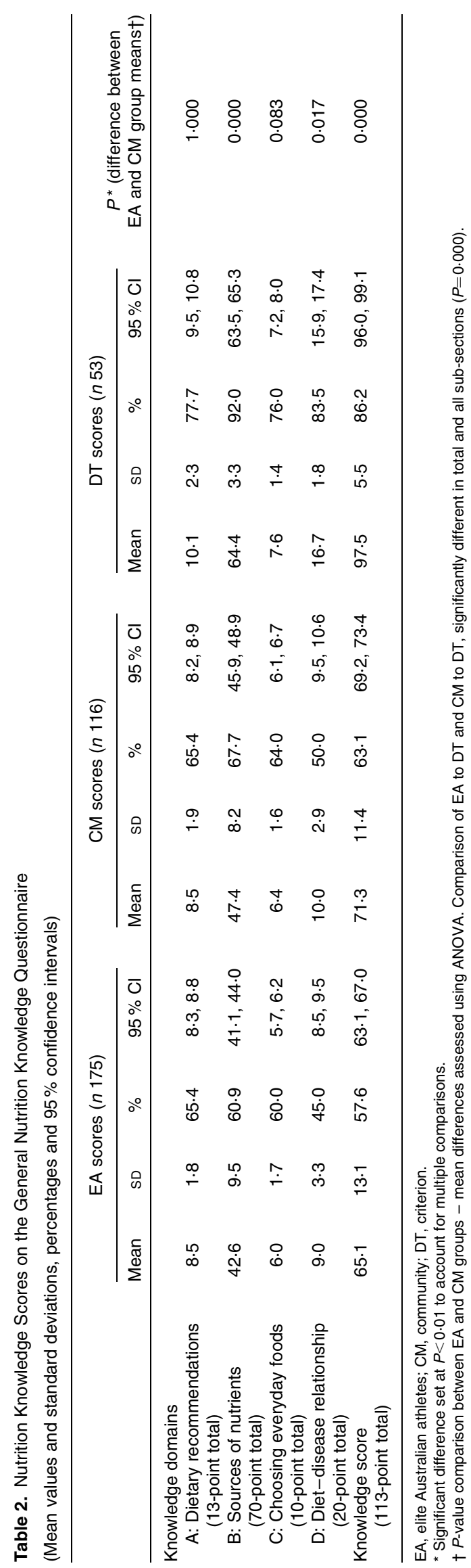


section $\mathrm{B},-8.5 \%$ for section $\mathrm{C},-18.3 \%$ for section $\mathrm{D}$ and $-8.7 \%$ for total score.

\section{Analysis of potential confounders}

After adjusting for age, sex, level of education and living situation, there was no effect of membership to EA or CM group on total nutrition knowledge in the GNKQ. Results indicate that older females had a higher total knowledge score than other participants, with younger male athletes scoring lowest. Despite the significance of age $(P=0.036)$ and sex $(P=0.016)$, these parameters only explained $6 \%$ of the variance between total knowledge score for EA and CM groups. Examination of the relationship between athletic participation and physical activity did not demonstrate a significant effect of athletic calibre $(P=0 \cdot 116)$ on total nutrition knowledge score in EA, nor did whether the participants were sufficiently active $(P=0.719)$ in CM. There was no significant effect of first language $(P=0 \cdot 151)$ or country of birth $(P=0.990)$ on total knowledge score in the EA and $\mathrm{CM}$ groups. Furthermore, there were no significant differences in nutrition knowledge between team or individual sports for national, junior or open international representatives. Analysis of state-level athletes was not conducted, as there were only three participants engaged in individual sports at state level.

Similar results were obtained in the R-GNKQ; however, sex was found to have a highly significant effect $(P=0.003)$, with age reduced to borderline significance $(P=0.053)$ on total nutrition knowledge score, after adjusting for level of education and living situation, as used for the GNKQ model. Sex and age explained a small but greater proportion (12.3\%) of the variance between $\mathrm{EA}$ and $\mathrm{CM}$ on the R-GNKQ. Fitting the model with only age and sex demonstrated that the older the respondent is, the higher their score. Even when age was controlled for in the model, males continued to score significantly less than females $(P=0.001)$. Older females were also found to have the highest total knowledge score on the R-GNKQ. In the EA and CM groups, there were no significant effects of first language $(P=0 \cdot 185)$ or country of birth $(P=0 \cdot 147)$. In contrast to the GNKQ, athletic calibre was found to have a significant effect $(P=0.029)$ on overall nutrition knowledge score in the revised version when adjusted for age. Athletes at open international level scored significantly higher than national $(P=0.036)$ or state representatives $(P=0.003)$. However, there were no significant differences in nutrition knowledge between team and individual sports for national, junior or open international representatives. Engaging in a sufficient level of physical activity for $\mathrm{CM}$ was again not found to have a significant effect $(P=0 \cdot 691)$ on total knowledge score.

\section{Psychometric evaluation of the General Nutrition Knowledge Questionnaire and re-examined General Nutrition Knowledge Questionnaire}

Table 4 presents the psychometric evaluation (internal consistency and test-retest measure) from a previous Australian study ${ }^{(23)}$ and the present study. The Cronbach- $\alpha$ (measure of 
Table 4. Internal consistency and correlation coefficient for Australian sample and present study

\begin{tabular}{|c|c|c|c|c|c|c|}
\hline & \multicolumn{2}{|c|}{$\begin{array}{c}\text { Australian sample } \\
\text { (n 156) - Hendrie et al. }\end{array}$} & \multicolumn{2}{|c|}{$\begin{array}{c}\text { GNKQ }(n 344)-\text { present } \\
\text { study (2010) }\end{array}$} & \multicolumn{2}{|c|}{$\begin{array}{c}\text { R-GNKQ ( }(n 344)-\text { present } \\
\text { study (2010) }\end{array}$} \\
\hline & $\begin{array}{c}\text { Internal } \\
\text { consistency* }\end{array}$ & $\begin{array}{l}\text { Correlation } \\
\text { coefficient }\end{array}$ & $\begin{array}{c}\text { Internal } \\
\text { consistency }\end{array}$ & $\begin{array}{l}\text { Correlation } \\
\text { coefficient }\end{array}$ & $\begin{array}{c}\text { Internal } \\
\text { consistency }\end{array}$ & $\begin{array}{l}\text { Correlation } \\
\text { coefficient }\end{array}$ \\
\hline \multicolumn{7}{|l|}{ Knowledge domain } \\
\hline A: Dietary recommendations & 0.53 & $0.37 \dagger$ & 0.56 & $0.64 \ddagger$ & 0.55 & $0.58 \ddagger$ \\
\hline B: Sources of nutrients & 0.88 & $0.85 \ddagger$ & 0.91 & $0.88 \ddagger$ & 0.91 & $0.89 \ddagger$ \\
\hline C: Choosing everyday foods & 0.55 & $0.75 \ddagger$ & 0.34 & 0.37 & 0.40 & $0.43 \dagger$ \\
\hline D: Diet-disease relationship & 0.73 & $0.74 \ddagger$ & 0.86 & $0.94 \ddagger$ & 0.86 & $0.90 \ddagger$ \\
\hline Nutrition knowledge score & 0.92 & $0.87 \ddagger$ & 0.93 & $0.92 \ddagger$ & 0.94 & $0.92 \ddagger$ \\
\hline
\end{tabular}

GNKQ, General Nutrition Knowledge Questionnaire; R-GNKQ, re-examined General Nutrition Knowledge Questionnaire.

* Internal consistency measured using Cronbach- $\alpha$.

$\dagger$ Spearman rank correlation (two-tailed) $<0.05$.

$\ddagger$ Spearman rank correlation (two-tailed) $<0.01$

internal consistency) for the overall nutrition knowledge scores was similar between the Australian published data $(0.92)$ and the present study in the GNKQ (0.93) and R-GNKQ (0.94). A Cronbach- $\alpha>0.80$ indicates good reliability, and this was the case for total score on the GNKQ and R-GNKQ and sections $\mathrm{B}$ and $\mathrm{D}$ in the present study. The Cronbach- $\alpha$ was lower and $<0 \cdot 80$ on sections $\mathrm{A}$ and $\mathrm{C}$, and this was also found to be the case for the published Australian data (Table 4).

The correlation coefficient for the test-retest analysis was highest for the overall nutrition knowledge score in both the GNKQ $(r 0.92, P<0.01)$ and R-GNKQ $(r 0.92, P<0.01)$, similar to the published Australian study $(r 0.87, P<0.01)^{(23)}$. The correlation coefficient was lowest for section $\mathrm{C}$, choosing everyday foods, in both the GNKQ and R-GNKQ (Table 5). The correlation coefficient of the EA group in section $\mathrm{C}$ of the GNKQ was poor ( $r$ 0.01). On further investigation, it was found that two of the EA participants scored significantly higher on their second attempt at the GNKQ (approximately $25 \%$ higher than initial attempt). Further analysis with these two participants excluded resulted in a substantially improved correlation coefficient for section C on the GNKQ in both EA $(r$ 0.502) and combined group analysis $(r 0.600$, $P<0.01)$. The highest correlation coefficient for the combined sample was for section D, diet-disease relationship, in both versions of the instrument (Table 5).

\section{Discussion}

The present study aimed to evaluate general nutrition knowledge in athletes using a previously validated instrument to benchmark scores against a non-athlete community group of similar age and a dietetic-trained cohort possessing a high degree of knowledge and expertise in nutrition. The inclusion of DT enabled EA and CM scores to be benchmarked against individuals expected to attain a high, if not perfect, score on the instrument. Considerable improvements in total and subsection scores were observed in the R-GNKQ in comparison to the GNKQ.

Knowledge of dietary guidelines (section A) was completed with the greatest accuracy (approximately $70 \%$ ) for both $\mathrm{EA}$ and $\mathrm{CM}$ and the smallest difference between DT (approximately 10\% less) compared to the other sub-sections. This reflects results from an Australian community sample $e^{(23)}$ and college athletes from the USA ${ }^{(17)}$ using the same instrument. Both EA and CM poorly answered the question on recommending consumption of more, the same or less 'starchy foods'. Despite the dietary guidelines being based on a carbohydrate-rich, low-fat dietary approach, recent media attention surrounding high-protein low-carbohydrate diets may have caused uncertainty about consumption of carbohydrate-rich foods ${ }^{(10,11,23)}$. In athletes, sufficient intake of carbohydrate is important for performance as well as health

Table 5. Spearman rank correlation coefficient for test-retest reliability in General Nutrition Knowledge Questionnaire (GNKQ) and re-examined General Nutrition Knowledge Questionnaire (R-GNKQ)

\begin{tabular}{|c|c|c|c|c|c|c|}
\hline & \multicolumn{3}{|c|}{ GNKQ (113 items) } & \multicolumn{3}{|c|}{ R-GNKQ (ninety-six items) } \\
\hline & $\mathrm{DI}(n 11)$ & $\mathrm{EA}(n 17)$ & Combined ( $n$ 28) & $\mathrm{DI}(n 11)$ & $\mathrm{EA}(n 17)$ & Combined ( $n$ 28) \\
\hline \multicolumn{7}{|l|}{ Knowledge domain } \\
\hline A: Dietary recommendations & $0.78^{*}$ & $0.52 \dagger$ & $0.64^{\star}$ & 0.52 & $0.56 \dagger$ & $0.58^{*}$ \\
\hline B: Sources of nutrients & $0 \cdot 81^{*}$ & 0.46 & $0 \cdot 88^{\star}$ & $0.78^{*}$ & $0.53 \dagger$ & $0 \cdot 89^{\star}$ \\
\hline C: Choosing everyday foods & $0.66 \dagger$ & 0.01 & 0.37 & 0.29 & 0.35 & $0.43 \dagger$ \\
\hline D: Diet-disease relationship & 0.47 & $0.86^{*}$ & $0.94^{*}$ & 0.24 & $0.68^{*}$ & $0.90^{\star}$ \\
\hline Nutrition knowledge score & $0 \cdot 84^{\star}$ & $0.66 \dagger$ & $0.92^{*}$ & $0.79^{*}$ & $0.66^{\star}$ & $0.92^{*}$ \\
\hline
\end{tabular}

DI, dietetic interns; EA, elite Australian athletes.

* Spearman rank correlation (2-tailed) $<0.01$.

† Spearman rank correlation (2-tailed) $<0.05$. 
reasons ${ }^{(27)}$. There is a need to clarify key public health messages on carbohydrate-rich foods.

EA fared worse than CM on sources of nutrients (section B) in both versions of the instrument and choosing everyday foods (section C) in the R-GNKQ (all $P<0.005$ ). Knowledge of choosing everyday foods (section $\mathrm{C}$ ) assessed procedural nutrition knowledge. On the GNKQ, section $\mathrm{C}$ was the second lowest knowledge domain (EA: 60.0\%; CM: 64.0\%) for both EA and CM. Both groups performed better in the R-GNKQ, although a significant proportion (50\%) of items was excluded due to poor design and ambiguity. Lower scores in sections $\mathrm{B}$ and $\mathrm{C}$ potentially reflect the focus of the compulsory learning objectives in the Australian high school curriculum, which has a stronger focus on declarative knowledge, especially knowledge of dietary guidelines rather than of the role of specific nutrients ${ }^{(28)}$.

Low exposure to food purchasing and preparing skills due to time constraints ${ }^{(4)}$ imposed by school and training commitments probably affected procedural nutrition knowledge needed for section $\mathrm{C}$, especially in the EA cohort. Incorporating sufficient procedural nutrition knowledge in the mandatory school curriculum would probably improve this component. The specific link between procedural nutrition knowledge, eating habits and lifestyle diseases deserves future research attention ${ }^{(2)}$.

Knowledge of diet-disease relationships (section D) was poor, with scores approximately $50 \%$ or less in both EA and $\mathrm{CM}$ groups. This section also had the greatest marked difference to the DT cohort, with EA scoring approximately $50 \%$ and $\mathrm{CM}$ about $35 \%$ lower than DT. Poor scores in this domain may be because participants were young (approximately $90 \%$ aged under 25 years), and the impact of diet on health may seem less relevant at this life stage ${ }^{(2)}$. This section of the instrument has been consistently poor in all community samples completing the GNKQ. The Australian community sample also scored about $50 \%{ }^{(23)}$, despite the cohort being comprised of predominantly older, tertiary-educated females. US female athletes scored in a similar range for diet-disease knowledge ${ }^{(24)}$. When the GNKQ was validated in the UK, knowledge of diet-disease relationships was poor with the non-nutrition-educated sample scoring approximately $35 \%{ }^{(11)}$. Although an understanding of how diet affects health would seem a critical factor in motivating healthier food choices ${ }^{(2,29)}$, participant interest may have waned by this stage ${ }^{(30)}$ of the questionnaire. For athletes, questions on understanding the impact of diet on performance may be more pertinent.

Consistent with the findings of other studies ${ }^{(3,10,11)}$, sex and age significantly influenced nutrition knowledge in EA and CM on the GNKQ and R-GNKQ. Older female participants scored highest and younger male participants lowest. Despite statistical significance, age and sex still only explained a small proportion of the variance in total knowledge score on both the GNKQ (6\%) and the R-GNKQ (12.3\%). In an Australian community sample, Hendrie et $a l .{ }^{(10)}$ found that age $(P<0 \cdot 01)$, employment status $(P<0 \cdot 01)$, highest level of education $(P<0.01)$ and sex $(P<0.04)$ had significant independent effects on nutrition knowledge ${ }^{(10)}$. These variables explained $40 \%$ of the variance in nutrition knowledge scores $^{(10)}$. Similarly, in the UK, Parmenter and colleagues ${ }^{(3,11)}$ found that male participants scored significantly lower in the $\mathrm{GNKQ}^{(11)}$, with sex $(P<0 \cdot 01)$, level of education $(P<0.01)$, occupational social class $(P<0.01)$ and marital status $(P<0.05)$ explaining $22 \%$ of the variance in knowledge scores $^{(3,11)}$. Lack of interest in nutrition has been proposed to be a contributing factor as to why males consistently score lower than females on nutrition knowledge ${ }^{(11)}$. Similarly, younger participants constantly score lower than middle-aged participants, which may potentially be attributed to the notion that nutrition knowledge is less relevant to the younger participants at this particular stage of $\operatorname{life}^{(3,10,11)}$. The positive association of nutrition knowledge and higher education and SES status is likely supported by the capacity to use written material to gain nutrition knowledge and implement favourable lifestyle behaviours ${ }^{(11)}$. Wardle and colleagues found that higher levels of education were significantly associated with better fruit intake, and those with higher SES had higher vegetable and lower fat intake ${ }^{(3)}$.

As the participants in both EA and CM cohorts were young, many still in high school living at home, it is likely that the responsibility for the majority of food purchases and preparation rests with the parents or guardians and not with the participants themselves. That being said, the younger female participants still scored significantly higher than the males, and this is evidence that even during late adolescence, knowledge in nutrition is biased by sex. Although $70 \%$ of the CM (Scouts) population targeted were male, $64.1 \%$ of respondents in this group were female. A higher proportion of female respondents were also recruited from the Faculty of Arts. A low male participation rate may reflect less interest or perceived competence in nutrition. Interest in the study of nutrition and working professionally as a dietitian is female dominated. Data from 1991-2005 found that $90.9 \%$ of dietitians in Australia were women and $9 \cdot 1 \%$ were men ${ }^{(31)}$.

Living situation was not found to have a significant effect on total knowledge score. As most of the EA and CM participants were young and living at home, with parents, a guardian or family, there was an insufficient range of participant living settings to thoroughly examine the impact of this parameter. Similarly, level of education did not significantly impact total knowledge score, but as with living situation, EA and CM samples were either at high school or tertiary educated, with most of the high school students completing the final two years (11 and 12), indicative of higher SES. The CM (Scouts) group were invited to participate as a means of obtaining participants from a range of SES and education levels. Unfortunately, participants (other than school students) were all tertiary educated.

In the R-GNKQ, athletes of higher calibre (open international) had a greater total nutrition knowledge score than national- or state-level athletes, even when adjusted for age. Superior nutrition knowledge in high-calibre athletes could be attributable to greater interest in nutrition, potentially driven by the pressure to exhaust opportunities to excel at the highest sports levels. Many open international athletes have greater interaction with dietitians, through group 
education, individual consultations to optimise training or competition nutrition strategies or during touring, when dietitians may travel as part of the sports science team ${ }^{(4,32)}$.

Evaluation of the GNKQ in the present study confirmed the instrument as valid and reliable. The psychometric evaluation of the GNKQ in the present study demonstrated similar coefficients and correlations to the previous Australian study in a community sample ${ }^{(10)}$ and the initial study validating this instrument in a UK community sample ${ }^{(22)}$. The correlation coefficients for test-retest on overall nutrition knowledge were high in both the GNKQ $(r 0.92, P<0.01)$ and R-GNKQ ( $r 0.92, P<0.01$ ), indicating good reliability for the instrument. An expert panel assisted in the removal of items where wording was ambiguous or the designated answer was not the only correct or best option. This resulted in elevated scores for the three cohorts in all sub-sections and total score. Future work on the instrument would seem relevant, particularly to redraft some of the items in section $\mathrm{C}$ (choosing everyday foods).

There are several limitations of the present research. Convenience samples were used, potentially reducing representativeness of the respective groups. The EA were slightly younger than CM when assessed by mean age, and a high proportion of EA were still at school and living in a home/ family situation. This probably influenced their responsibility and role with food selection and preparation, which would be expected to make an impact on nutrition knowledge. Living situation was not found to significantly affect nutrition knowledge, but with the sample heavily weighted to school age participants, there was insufficient spread of living situations for adequate assessment. Attempts to recruit male participants in the CM sample from a wider education level were unsuccessful, with a predominantly female, tertiary-educated cohort responding. This was despite the incentive of movie tickets for randomly selected respondents on instrument completion. The strong response from females and the low recruitment of males suggests potential selective uptake of males more confident in nutrition knowledge. Knowledge scores for males in the present study may not be universal to the large proportion of male non-respondents. Because EA were younger, there was less representation of high-calibre, international-level athletes. As higher athletic calibre was associated with better nutrition knowledge, future studies would need to aim for a more consistent recruitment from each competitive level. Insufficient numbers from team or physique sports meant that they could not be evaluated for nutrition knowledge.

Strengths of the present study include the use of a previously well-validated instrument using appropriate food terms for the Australian setting supported by additional psychometric analysis in the sample cohorts of the present study. Recruitment of a similar aged community group and a criterion sample with dietetic training provided context to the athlete nutrition knowledge scores. Content validity of the instrument was assessed by a panel of experienced dietitians and nutrition researchers that resulted in revision of the instrument (R-GNKQ), which appeared to be more sensitive than the GNKQ in teasing out significant differences. Measurement of known (e.g. age, sex and level of education) and potential confounders (e.g. living situation, level of physical activity and ethnicity) of nutrition knowledge in the sample has been a frequent omission in many of the previous studies of nutrition knowledge in athletes ${ }^{(5)}$. In the EA sample, measurement of athletic calibre and type of sport allowed assessment of these factors.

\section{Conclusions and future directions}

The present study assessed the level of general nutrition knowledge in elite Australian athletes compared to a community sample of similar age, benchmarked against dietetictrained cohort. The level of nutrition knowledge in both the EA and CM cohorts was strong for dietary recommendations and poor for diet-disease relationships with knowledge of sources of nutrients in food and choosing everyday foods between these two constructs. The results of the present study suggest that knowledge should be improved, particularly that pertaining to diet-disease relationships and procedural knowledge associated with choosing everyday foods.

The total knowledge score of EA was moderately, but significantly, lower than CM. EA were younger and had a higher proportion of males. These demographic factors had a significant negative impact on knowledge scores, but only explained a small proportion of the variance. Level of education, living situation, ethnicity and sport type were not found to significantly impact nutrition knowledge, but adequate representation of these factors was not achieved. Athletes of international calibre possessed higher levels of knowledge (age adjusted) than national or state representative peers.

Future studies need to recruit larger numbers of participants with wider, equal representation so that the effect of age, sex, education and SES on nutrition knowledge can be investigated further. Wider representation of sports-specific factors including, athletic calibre, team, individual and physique-based sports is recommended. Finally, the impact of general and sports-specific nutrition knowledge on dietary intake behaviours has not been adequately assessed, and this association is important for determining and then advocating the provision of adequate nutrition education to elite athletes. Validated instruments to measure sports nutrition knowledge are required.

\section{Acknowledgements}

The present research was supported by a National Elite Sports Council grant from the Australian Sports Commission for the collection of athlete data. The authors thank the New South Wales Institute of Sport, Queensland Academy of Sport, Victorian Institute of Sport, the South Australian Sports Institute, Collaroy Surf Life saving club, the Bulldogs NRL club, the University of Sydney, and in particular, the Faculty of Arts and Science, Scouts New South Wales and Australian Capital Territory and the Accredited Practicing Dietitians who participated in the study. The authors would also like to acknowledge Gillian Anne Hendrie for the use of the instrument in the present research. J. K. S. collected the CM and DT data, 
analysed the EA, CM and DT data and wrote the majority of the paper. S. E. H. collected the EA data, assisted with data analysis and provided input into the development of the paper. J. A. G. assisted with developing the study method and provided support and feedback. T. P. provided guidance and assistance with the statistical analysis. G. S. D. assisted with developing the study method and provided support and feedback. H. T. O'C. was heavily involved in developing the study method, statistical analysis, development and writing of the paper, and provided support and feedback. The authors declare no conflict of interest.

\section{References}

1. American Dietetic Association, Dietitians of Canada and American College of Sports Medicine, et al. (2009) Joint Position Statement: nutrition and athletic performance. Med Sci Sports Exerc 41, 709-731.

2. Worsley A (2002) Nutrition knowledge and food consumption: can nutrition knowledge change food behaviour? Asia Pac J Clin Nutr, 11, Suppl., S579-S585.

3. Wardle J, Parmenter K \& Waller J (2000) Nutrition knowledge and food intake. Appetite 34, 269-275.

4. Heaney S, O'Connor H, Naughton G, et al. (2008) Towards an understanding of the barriers to good nutrition for elite athletes. Int J Sports Sci Coach 3, 391-401.

5. Heaney S, O'Connor H, Gifford J, et al. (2011) Nutrition knowledge in athletes: a systematic review. Int J Sport Nutr Exerc Metab 21, 248-261.

6. Axelson ML, Federline TL \& Brinberg D (1985) A meta-analysis of food-related and nutrition-related research. $J$ Nutr Educ 17, 51-54.

7. Obayashi S, Bianchi J \& Song WO (2003) Reliability and validity of nutrition knowledge, social-psychological factors, and food label use scales from the 1995 Diet and Health Knowledge Survey. J Nutr Educ Behav 35, 83-92.

8. Nestle M, Wing R, Birch L, et al. (1998) Behavioural and social influences on food choice. Nutr Rev 56, 50-64.

9. Dallongeville J, Marecaux N, Cottel D, et al. (2000) Association between nutrition knowledge and nutritional intake in middle-aged men from northern France. Public Health Nutr 4, 27-33.

10. Hendrie GA, Coveney J \& Cox D (2008) Exploring nutrition knowledge and the demographic variation in knowledge levels in an Australian community sample. Public Health Nutr 11, 1365-1371.

11. Parmenter K, Waller J \& Wardle J (2000) Demographic variation in nutrition knowledge in England. Health Educ Res 15, 163-174.

12. Sapp SG \& Jensen HH (1997) Reliability and validity of nutrition knowledge and diet-health awareness tests developed from the 1989-1991 diet and health knowledge surveys. J Nutr Educ 29, 63-72.

13. Turrell G \& Kavanagh AM (2006) Socio-economic pathways to diet: modelling the association between socio-economic position and food purchasing behaviour. Public Health Nutr 9, 375-383.
14. Hamilton GJ, Thomson CD \& Hopkins WG (1994) Nutrition knowledge of elite distance runners. NZ J Sports Med 22, 26-29.

15. Worme JD, Doubt TJ, Singh A, et al. (1990) Dietary patterns, gastrointestinal complaints, and nutrition knowledge of recreational triathletes. Am J Clin Nutr 51, 690-697.

16. Douglas PD \& Douglas JG (1984) Nutrition knowledge and food practices of high school athletes. J Am Diet Assoc 84, 1198-1202.

17. Dunn D, Turner LW \& Denny G (2007) Nutrition knowledge and attitudes of college athletes. Sports J 10, 1-5.

18. Harrison J, Hopkins WG, MacFarlane DJ, et al. (1991) Nutrition knowledge and dietary habits of elite and non-elite athletes. Aust J Nutr Diet 48, 124-127.

19. Werblow JA, Fox HM \& Henneman A (1978) Nutrition knowledge, attitudes and food patterns in women athletes. J Am Diet Assoc 73, 242-245.

20. Wiita B, Stombaugh I \& Buch J (1995) Nutrition knowledge and eating practices of young female athletes. $J$ Physic Educ Recr Danc 66, 36-41.

21. Barr SI (1986) Nutrition knowledge and selected nutritional practices of female recreational athletes. J Nutr Educ 18, 167-174.

22. Parmenter K \& Wardle J (1999) Development of a general nutrition knowledge questionnaire for adults. Eur J Clin Nutr 53, 298-308.

23. Hendrie GA, Cox DN \& Coveney J (2008) Validation of the general nutrition knowledge questionnaire in an Australian community sample. Nutr Diet 65, 72-77.

24. Raymond-Barker P, Petroczi A \& Quested E (2007) Assessment of nutritional knowledge in females athletes susceptible to the Female Athlete Triad syndrome. J Occup Med Toxicol 2, 10.

25. Kline P (2000) The Handbook of Psychological Testing, 2nd ed. London: Routledge.

26. Australian Institute of Health and Welfare (2003) Indicators of health risk factors, the AIHW view. http://www.aihw. gov.au/publications/index.cfm/title/9349

27. Heaney S, O'Connor H \& Gifford J (2010) Comparison of strategies for assessing nutritional adequacy in elite female athletes' dietary intake. Int J Sport Nutr Exerc Metab 20, $245-256$.

28. New South Wales Government (2007) Personal development, health and physical education years 7-10 syllabus. http://www.boardofstudies.nsw.edu.au/syllabus_sc/pdf_ doc/pdhpe-7-10-syllabus.pdf

29. Grunert KG, Brunso K \& Brisp S (1993) Food-related Life-styles: Development of a Cross-culturally Valid Instrument for Market Surveillance. Mapp Working Paper no. 12. Aarhus: The Aarhus School of Buisness.

30. Burchell B \& Marsh C (1992) The effect of questionnaire length on survey response. Qual Quant 26, 233-244.

31. Brown L, Capra S \& Williams L (2006) Profile of the Australian dietetic workforce: 1991-2005. Nutr Diet 63, 166-178.

32. Cummings N, Pelly F, Dang V, et al. (2010) Providing meals for athletic groups. In Clinical Sports Nutrition, 4th ed., p. 686. Sydney: McGraw-Hill. 